\title{
Automation of Embodied Energy Estimation Using Programming Tool
}

\author{
Abhijeet D. Patil ${ }^{\mathrm{a}}$, Dr. A.C.Attar ${ }^{\mathrm{b}}$ \\ ${ }^{a}$ Student, Rajarambapu.Institute of.Technology,Rajaramnagar(MH), India. \\ ${ }^{b}$ Professor, Rajarambapu.Institute of.Technology, Rajaramnagar(MH), India.
}

\begin{abstract}
Worldwide Building consumes annually about 30-40\% of primary energy in construction, operation and maintenance. A massive part of this energy is consumed in fabric activity alone. Nearly two million residential buildings for economic weaker section alone are required to be built annually in India, apart from offices, commercial, public and industrial buildings. Estimation of embodied energy is quite difficult due to complex calculations. In case of raw material energy, it may be easy. But for thermal, electric and transportation, it may be difficult due to the relationship between fuel used and the capacity of vehicle. Also the electricity required for particular amount of material cannot be predicted accurately. So there is need of some computing tool to directly calculate the embodied energy (E.E.) of building components. This paper seeks to cover the brief information about the programming tool (software) of calculating embodied energy. This software is designed to complete the all requirements of the user. All the tabs and functions are so synchronized that, it can be handled by the person who is not familiar to the topic. The E.E. of basic materials should be given to the software and by giving quantity of component and proportion; it gives the E.E. of component in MJ. Also the energy conversion of $J$ to $K J, M J, G J$, and TJ should be given in this software. The all other information about the E.E. like carbon content, power conversion etc. is fitted into the software. The window named 'Project' is given to cluster the all components in any building. In case of R.C.C. work, all the sub components are differentiated separately. Because the steel required for sub components are different.
\end{abstract}

Keywords: Embodied Energy, programming tool;

\section{Introduction}

Analysis has shown that buildings are one of the largest sources of greenhouse gas emissions, energy usage and other associated emissions globally. Although during the whole life cycle (40 years) the energy and (most) emissions of the utilization phase accounts for $80-90 \%$ of the impact of the building sector, the assessment of embedded impacts still needs to be analysed, especially when materials with a high productionassociated impact are being used. This is particularly pertinent in the case where more sustainable (operational) housing is being sought, as the embedded impacts can be significant. In the design of "greener" buildings, it is important not to neglect the embodied impacts e especially as the buildings operational impacts are dramatically decreased, the significance of embodied impacts becomes higher in a relative sense. As with most multicomponent products, the volume of material use is not always an indicator of its environmental contribution. Building material that is highly significant to the total weight may not be a significant contributor to the total building impacts e for instance one analysis showed concrete contributing more than $74 \%$ only accounted $14 \%$ of total life cycle impacts, while building service components accounted approximately $27 \%$ of total life cycle impacts but less than $2 \%$ of the weight. As one of the most populous nations in the world, and significantly, one of the largest developing nations in the tropics, Indonesia is an ideal case study for examining the potential for mitigating environmental impacts of buildings. Furthermore, in many rapidly developing countries e such as Indonesia there is a tension between policy aimed at economic development, environmental protection through operational standards and a social preference to move away from traditional materials towards modern building materials. This study examines the potential impacts or benefits of the move from more traditional clay-based housing to concrete-based housing from the perspective of the embodied impacts. Debate continues about the boundaries that should be applied to calculating embodied energy. Commonly, the most influential components of embodied energy are those bounded by the cradle to gate approach, that is, all the energy required to deliver the product to the gate of the factory ready for transport to the construction site. Even within an embodied energy calculation bounded "cradle to gate" the complexity of embodied energy could be extreme. For example, the energy used by the factory in the processing or manufacturing process may be easily identified, however what about the energy used by the employees: Transport Fuel (to and from work), Embodied energy of transport (to and from work), Energy of services (health, legal, accounting), Energy to produce food to feed the workforce (transport, agriculture, refrigeration) Any one point in the processing and manufacturing chain can be analysed in detail endless trail of energy calculations back to the stone age. With this in mind, it is important to remember the purpose of embodied energy calculations, it is to make informed decisions that lead to improvements in the 
way we use energy. At present, order of magnitude accuracy would generally satisfy this purpose. The approach discussed in this paper is built on this principal.

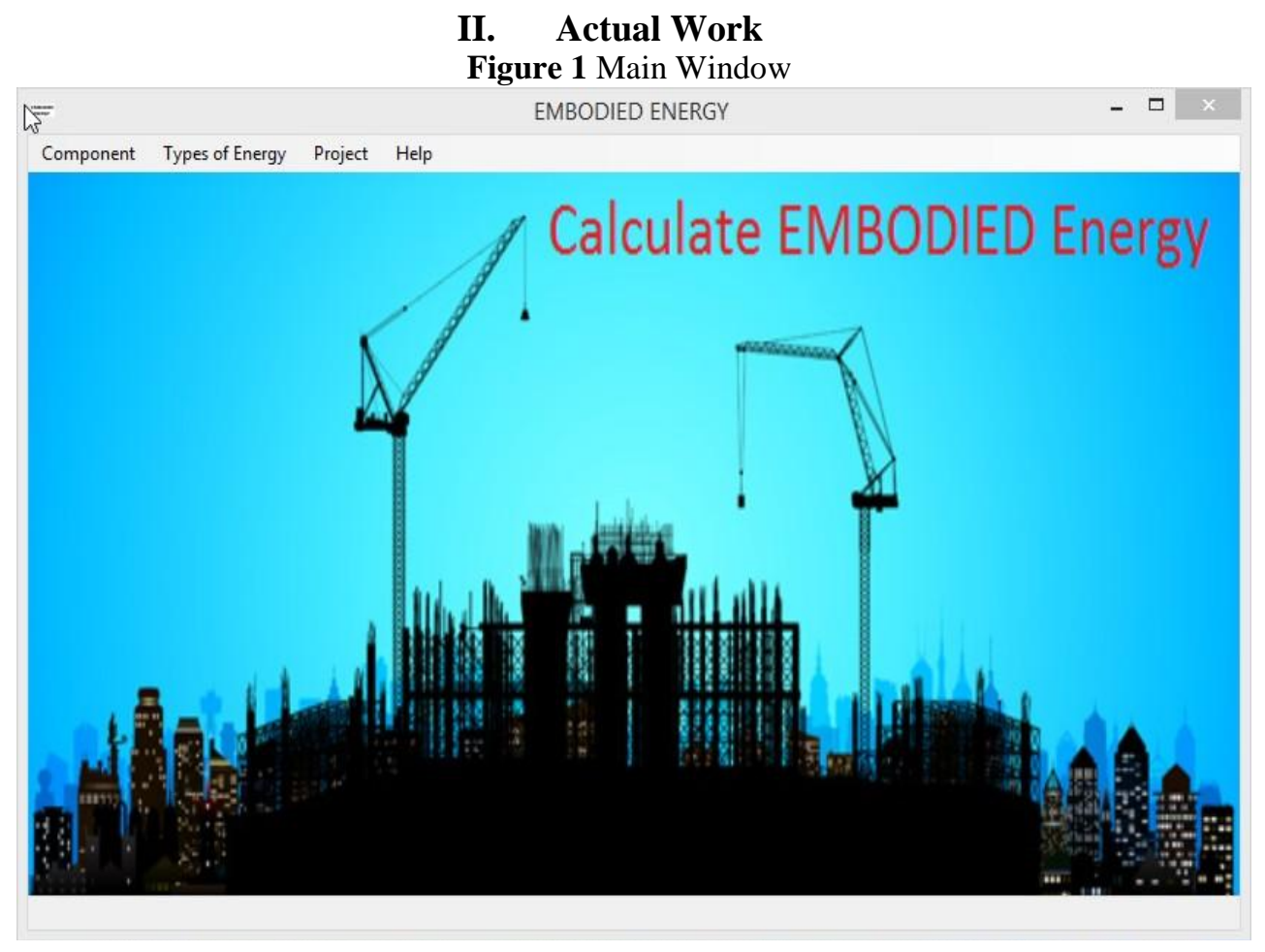

Software is designed in such a manner that all the parts of Embodied Energy Calculation should be covered. The software can be easily used by the person who doesn't know about the calculation. In this software, four tabs are added, viz. Component, Types of energy, Project, Help. He have to only collect the information about the quantity of component and the proportion of material. In software, all components of R.C.C. building should be covered. In case of some components, the E.E. of basic raw material is not available. So in such case, user has to enter the E.E., then software will calculate the Total energy. The three types of energies are added viz. Electrical, Thermal and Transportation. By adding required information like amount of fuel, electricity required, efficiency of vehicle etc., we will get the particular energy. The tab named 'Project' is added to compile all the data required for completion of any project. In this tab all components will see, we have to select required component and by adding all components total energy will come at the end. In 'Help' tab, all the require information for using software is given like conversion, energy of materials etc.

\subsection{Component:}

Total nine components are included in this tab namely brickwork, R.C.C., Excavation P.C.C., Backfilling, Doors and windows, plastering, flooring, painting.

\subsubsection{Brickwork:}

In brickwork, six types are added namely Burnt Brick, Fly Ash Brick, Hollow Block, Aerated Block, solid Concrete Block, and Soil Cement Block, Whose E.E. for particular block is fitted into the software. User can select any type of Brickwork. Then User has to select the size of Brick. Because size is different for all bricks. Then user can select the proportion of mortar. The E.E. of cement and sand is fitted into the software. After adding all these values, user has to click on button named 'Calculate Energy'. Then E.E. comes for 1 Cu.m. Brickwork for required type. Figure 2shows the all tabs included in 'Brickwork' window.

\subsubsection{R.C.C. work and P.C.C. Work:}

In R.C.C. work, total seven sub components are added namely footing, column, beam, lintel, slab, lift wall and sill whose Steel percentage should be $0.5 \%, 2 \%, 1.5 \%, 1 \%, 1 \%, 1 \%$ respectively. Other procedure is same for all sub components. User has to enter the quantity of work in Cu.M.and proportion of mortar. The E.E. of cement, sand and aggregate is already fitted into the software. Then by clicking on 'Calculate Energy' button, it gives E.E. for particular quantity in MJ. The procedure is same for P.C.C. work. Only the steel should not be added in this case. Figure 3 shows the all tabs included in 'R.C.C. Work' window. 


\subsubsection{Excavation and Backfilling:}

In 'Excavation' tab, excavated area has to be added in Cu.M. Then Required diesel should be added in litre. Then by clicking on 'Calculate energy' button, Diesel Energy comes for required quantity in MJ. Generally most of the vehicles requires diesel. So Calorific value of diesel(38.7MJ) has been added to the software. Same procedure should be followed for Backfilling process. Figure 4 shows the all tabs included in 'Excavation' window.

\subsubsection{Doors and Windows:}

In 'Doors and Window' tab, there are three sub components are added viz. wooden, iron and aluminium. The E.E.for these sub components per sq.m.has been added into the software. So user has to add the size of door/ window. Then by clicking on 'Calculate energy' Button, E.E. of component of required size should get. Figure 5 shows the all tabs included in 'Doors \& Windows' window.

\subsubsection{Plastering:}

In 'Plastering' tab, Quantity of plastering work and the proportion of mortar has to be added by the user in Cu.M.. The software will do the required calculation for dry mortar and fill up joints. The E.E. of raw materials should be fitted in the software. By clicking on 'Calculate Energy' button, user will get the E.E. in MJ for required quantity. Figure 6 shows the all tabs included in 'Plastering' window.

\subsubsection{Flooring:}

In 'Flooring' tab user has to add quantity of flooring in sq.m. The E.E. of various tiles is different. And collection of such data is a hard task. So E.E. of tiles has not been added in to the software. So the user has to enter the E.E. of appropriate tile of 1 sq.m.and the proportion of mortar. The software will calculate the dry quantity of materials. Then by clicking on 'Calculate Energy' button, user will get the E.E. of flooring work for required quantity in MJ. Figure 7 shows the all tabs included in 'Flooring' window.

\subsubsection{Painting:}

In 'Painting' tab user has to add quantity of painting in sq.m. The E.E. of various paint is different. And collection of such data is a hard task. So E.E. of paints has not been added in to the software. So the user has to enter the E.E. of appropriate paint of 1 sq.m. and the proportion of mortar. The software will calculate the dry quantity of materials. Then by clicking on 'Calculate Energy' button, user will get the E.E. of painting work for required quantity in MJ. Figure 8 shows the all tabs included in 'Painting' window.

\subsection{Types of energy:}

\subsubsection{Thermal:}

Thermal energy is the energy created by burning the fuel for any purpose. In this tab, user has to enter the amount of product for which fuel is burnt. Then user has to give the type of fuel. There are various fuels are used for burning. But mainly coal and wood are used. So these fuels are taken into consideration. Their calorific value has been added into the software. Then user can give the amount of fuel in $\mathrm{kg}$. Then by clicking 'Calculate Energy' button, Thermal energy will get for single product in MJ. Figure 9 shows the all tabs included in 'Thermal Energy' window.

\subsubsection{Electrical:}

Electric energy is the energy produced by electricity during the manufacturing of product. User has to enter the total electricity required for particular product in HP. Also total time required for completing the process has to be entered by the user. Software will do the required calculations. The required conversions has been added such that, $1 \mathrm{HP}=745.6 \mathrm{Watts}$ and $1 \mathrm{Watt}=1 \mathrm{Joule} / \mathrm{Sec}$. By clicking on 'Calculate Energy' button, Electric energy will get in MJ. Figure 10 shows the all tabs included in 'Electric Energy' window.

\subsubsection{Transportation:}

Transportation is the main source of energy in case of all the materials. Because raw material is collected from different places. So the transportation distance, type of vehicle, efficiency of vehicle and capacity of vehicle should be taken into consideration. So by calculating the diesel required for required quantity, the diesel energy can be calculated. User has to click on button 'Calculate energy', he will get the Transportation Energy in MJ. Figure 11 shows the all tabs included in 'Transportation Energy' window.

\subsubsection{Miscellaneous:}

There are miscellaneous products like rice husk brick, wood ash brick, baggas ash brick etc. whose energy cannot be calculated by the traditional methods. For such products, this tab is given. In this tab, type of 
material should be added by the user. Also the quantity of material, and all other required energies like transportation, electrical and thermal energy should be added by the user. So by clicking on 'Calculate Energy' button user will get the Embodied energy of appropriate material in MJ. Figure 12 shows the all tabs included in 'Miscellaneous' window.

\subsection{Project:}

All the data is compiled in this window named 'Project'. First column is 'Component' in which all the components will come by clicking on bottom arrow. Then by adding required information, the component energy will come which has to be added into the second column named 'Component Energy'. Third to fifth column will contain the electrical thermal and transportation energy respectively. The user has to add the required energy for particular component. Then by clicking on 'Calculate' button, E.E. will come for that component. The button 'Add component' has been added to add more components if required. By adding more components, Total Project Energy will display at the bottom of window. All of the required energy will be displayed to the user at the end of the project. This is the most important tab in all manners.Figure 13 shows the all tabs included in 'Project' window.

\subsection{Help:}

\subsubsection{Conversion:}

In 'Conversion' tab, Conversion of Joule to Kilo Joule, Mega Joule, Giga Joule, Tetra Joule is given. By adding value of Joule, automatically all other values comes. This is most important tab in all other tabs. Because in some cases, values are given in various other formats. So this conversion has a vital importance. Figure 14 shows the all tabs included in 'Conversion' window.

\subsubsection{Other Information:}

All other information like carbon content, Energy conversion, Power conversion, weight conversion is included in this window. While calculating E.E. these information may be required. Because in some cases energy is given for its weight; user require for the volume. So he must know about the important conversions. Figure 15 shows the all tabs included in 'Other Information' window.

\subsubsection{How to use Software:}

For new user, he must know about the various functions in the software. So this tab helps to know about the various tabs in software. Also the stepwise procedure is given in this tab to calculate E.E. There are three points are given in this tab namely components, types of energy and project. In each point, the information to calculate the E.E. for particular product or component is given. Figure 16 shows the all tabs included in 'How to use' window.

\subsubsection{Energy of Materials:}

In this tab, E.E. of basic materials is given, which is used for previous calculation. So it helps to cross check the all over calculation. The E.E. is given per component, per sq.m., per cu.m., per kg. These values are taken from research paper; some values are calculated manually. These values may change region wise. Also the methods may vary for calculation. There may be changes in values. These values are not standard values. As per author's opinion, these values are taken. Figure 17 shows the all tabs included in 'Energy of Materials' window.

\subsubsection{Add Item:}

In this tab, Items can be added. In this window, Item no. Description, Unit Name and Component Name are added. Item nos. is unique for every project. So it is important to mention it. Description contains the detailed explanation of the work carried out. Generally three types of units are used viz., CU.M. SQ.M. and R.M. Figure 19 shows the all tabs included in 'Add Item' window.

\subsubsection{Add Project:}

In this tab, new project can be added. The difference between the previous tab 'Project' is the project done by using this tab can be saved in PDF format. The name of project plot area, Built up area, total floors and description of buildinh should be filled by the user. Then only he has to select item no. The appropriate things will display automatically. Then by clicking Add button, the item will be added to the list. Figure 20 shows the all tabs included in 'Add Project' window.

\subsubsection{Project List:}

In this tab, the projects should display which was done previously. Only the user have to remember the project ID, then he can easily find the project. Then click on 'Generate Report' Button. The output will be the 
PDF file contains the energy description of the concern building. Figure 21 shows the all tabs included in 'Project List' window.

\section{Comparison Of Software With Manual Calculation}

In manual calculation, there may be some errors due to the complex calculations. In most of cases, whole to part calculation is required. So it is quite difficult manually. So software can do these calculations easily due to high capacity processor. Also it gives the more accuracy in calculations. In any project, it consists of various components. E.E. of each component consists of various energies like transportation, electrical, thermal. So calculation of these parts takes more manually. In other words, it is a time consuming activity. But in software, there is a window named "Project". It compiles all the data required for any project. User has to add only the components required for particular building. It calculates total energy required for that project. So software can save time required for the complex calculations. Due to automization, the complexity can be reduced. The formulae and the E.E. of basic materials can be already given to the software. So by giving the input to the software, E.E. can be calculated directly by the software. There is only matter of the values given to the software. If those are correct, then output will be the expected one. For the calculation of E.E. of any component, user can select any of the alternatives available in the market. So software is designed in such a way that, E.E. calculation of all components can do easily. In case of brickwork, burnt, fly ash, soil cement, hollow, aerated blocks are added. In case of doors and windows, wooden, iron and aluminium options are added. In case of flooring, there is a space to add the E.E. of tile of the selected option. So these important points are to be taken into care while designing the software. It should be designed in such a way that it should satisfy all needs about E.E.

In case of many projects, some materials are included; this cannot be categorized in any component. So for these materials, the window is provided named "Miscellaneous". So by using this window user can add miscellaneous materials. Bu E.E. of these materials has to calculate manually by user. Only compilations of all energies are available in the software. This software is useful for multistoried building where lots of components are included. In 'project' window, There is a calculate button from which it calculates total E.E. of particular component. Also there is a button namely 'Add Component' is provided. User can add infinite nos. of component by using this button. The total energy can be calculated and displayed at the bottom automatically.

In 'Help' tab, there is an option named 'Conversion' is available, by which we can convert from Joule to MJ, GJ, KJ, TJ. This is most important point in case of energy calculation. Because most of times values comes in decimal points. So it is better to express those values in other units. Also E.E. of basic materials is given in this tab. So new user can cross check the values calculated by software. Also the general information about E.E. is given in this tab. By using the window named 'How to use software', new user can know about the various functions in the software.
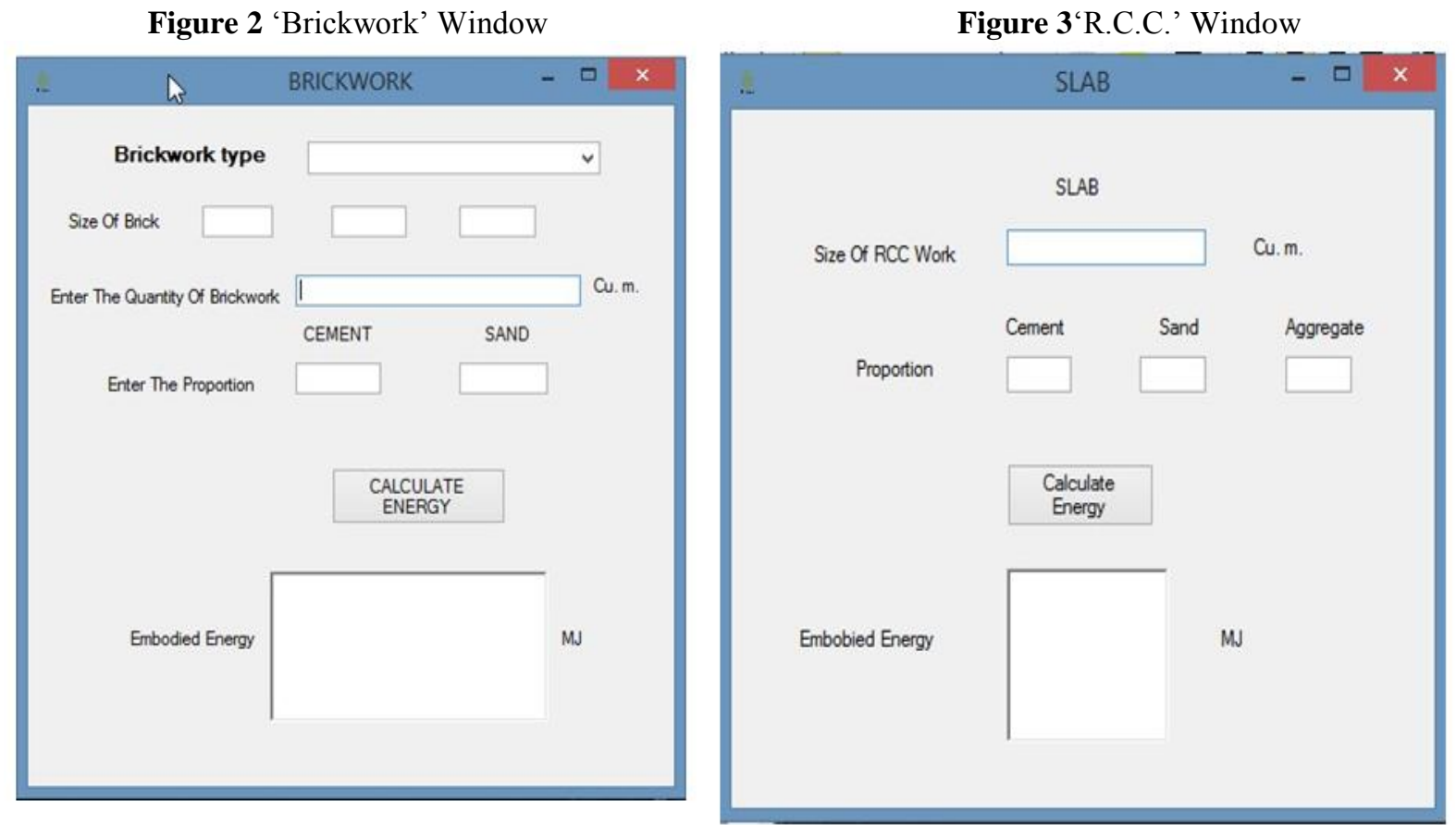
Figure 4 'Excavation' Window

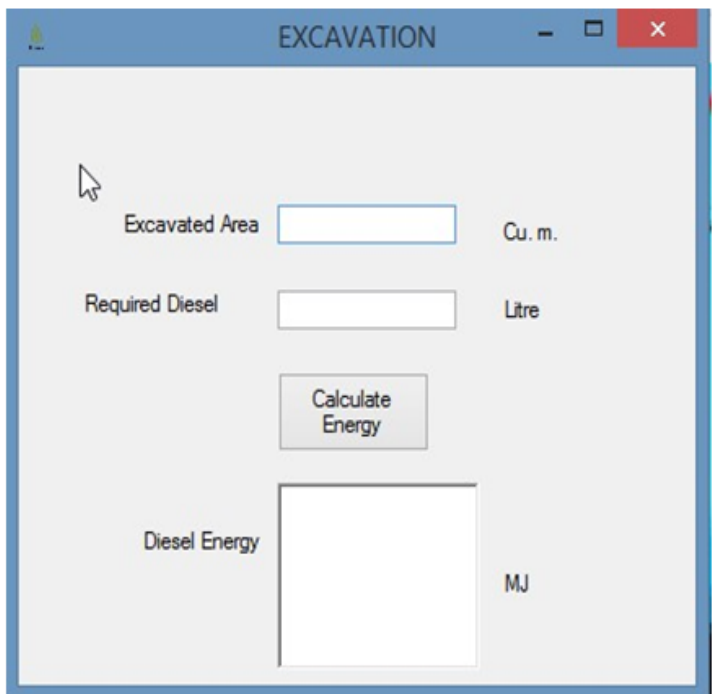

Figure 6 'Plastering' Window

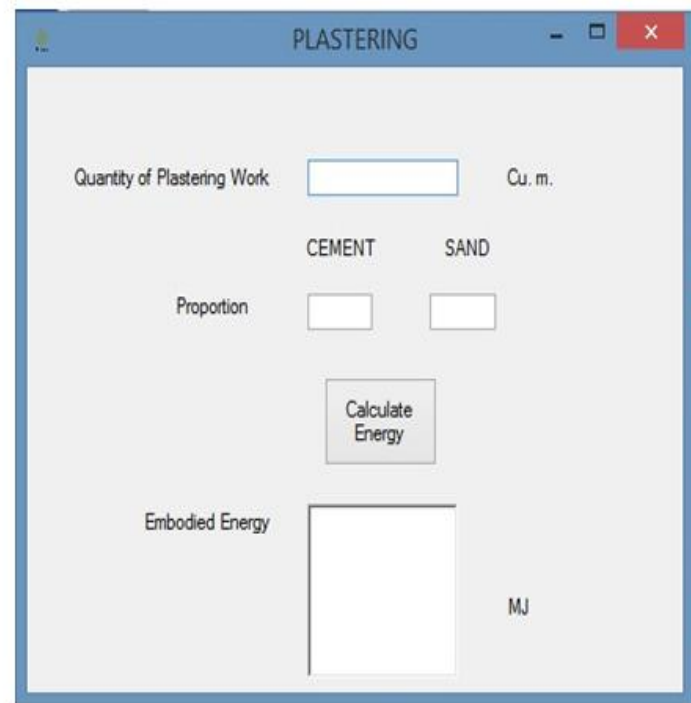

Figure 8 'Painting' Window

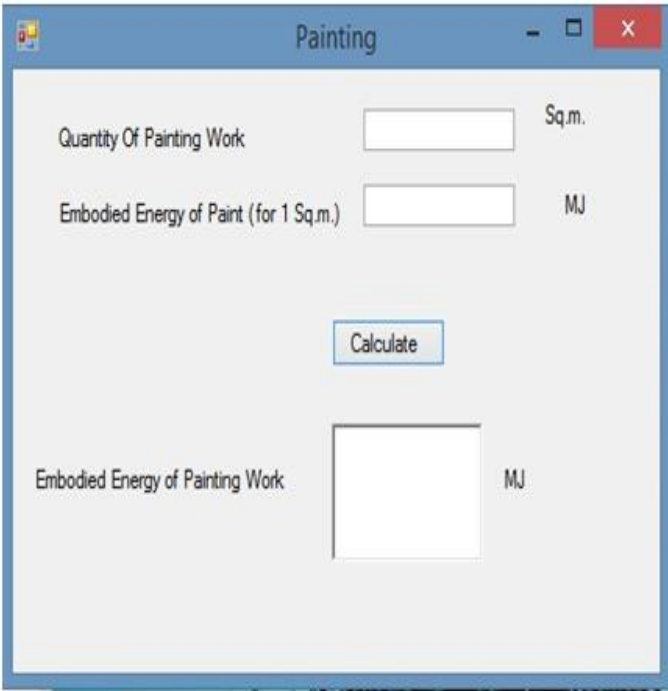

Figure 5 'Doors \& Window'

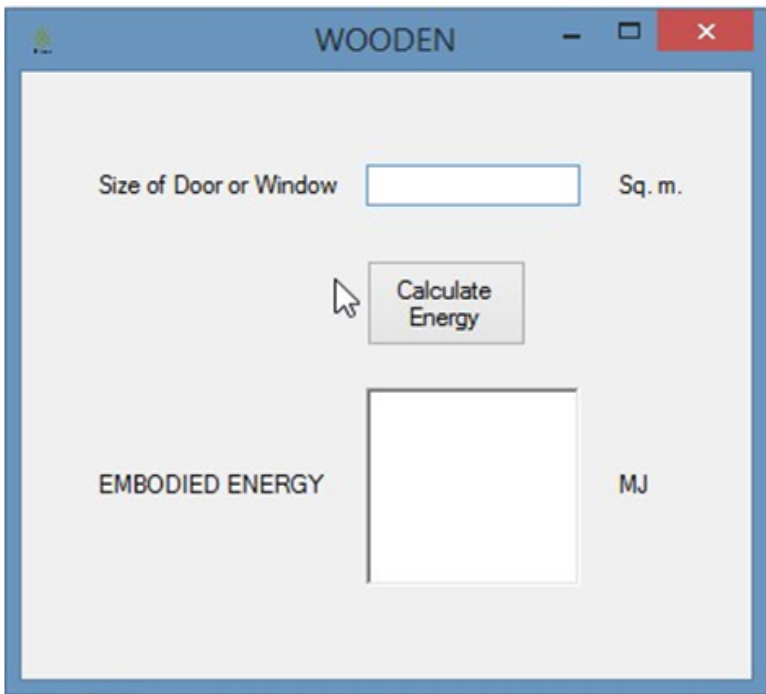

Figure 7 'Flooring' Window

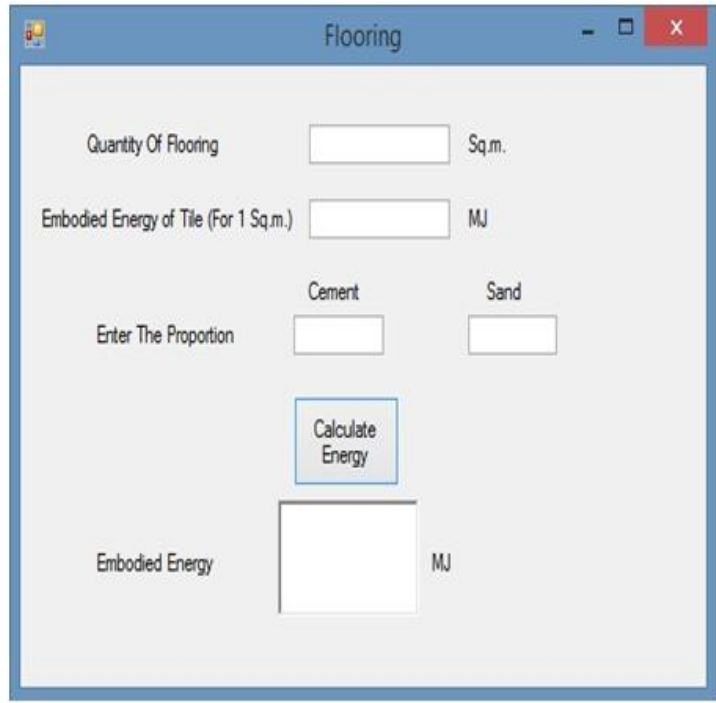

Figure 9 'Thermal' Window

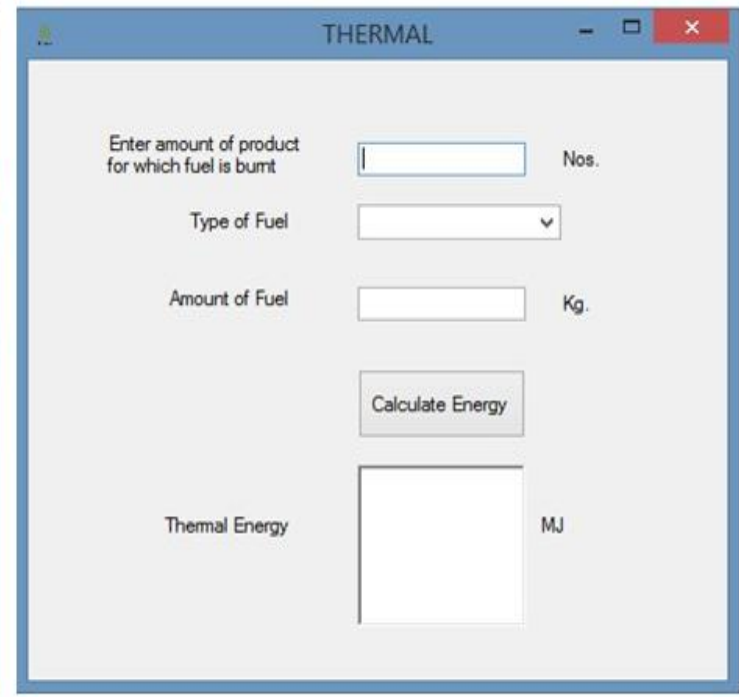


Figure 10 ‘Electrical Energy’ Window

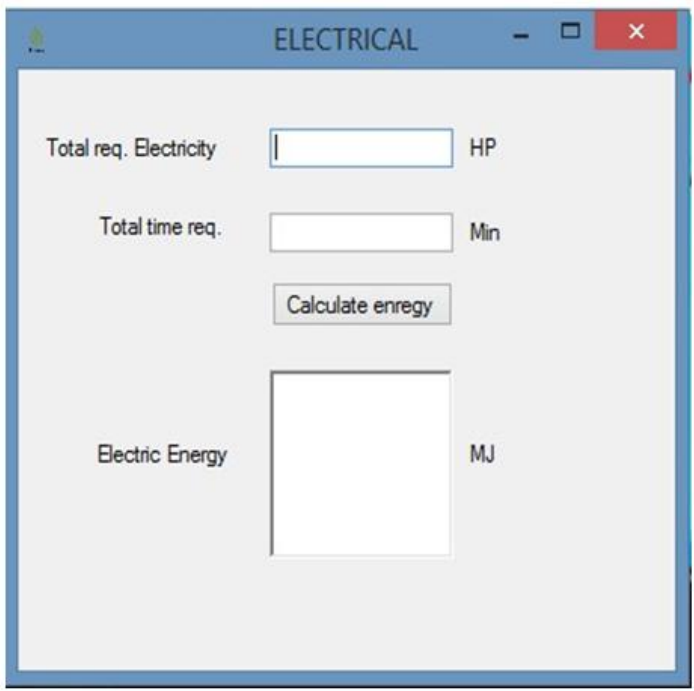

Figure 12 'Miscellaneous' Window

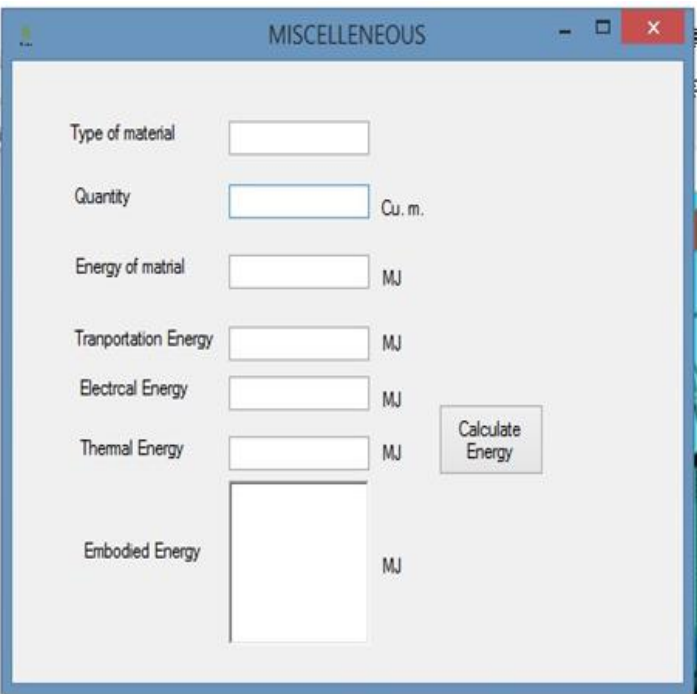

Figure 11 'Transportation energy' Window

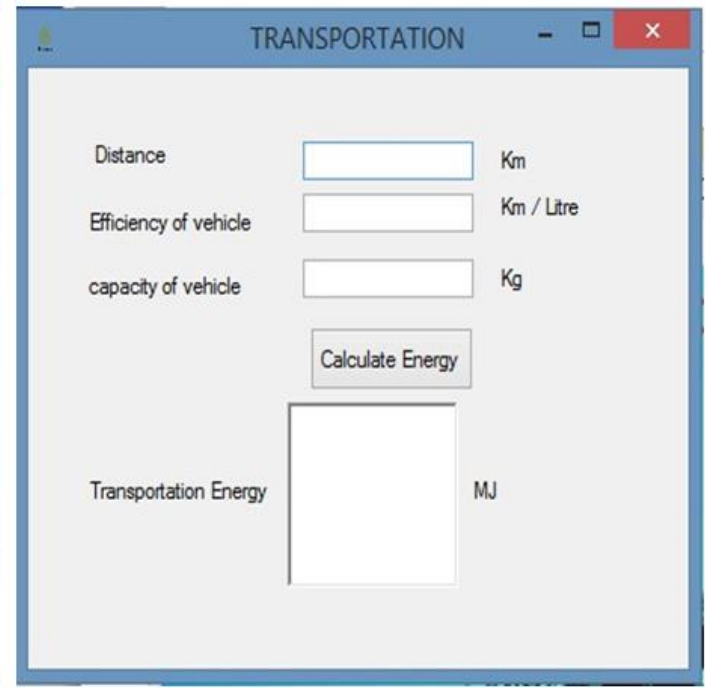

Figure 13 'Conversion’ Window

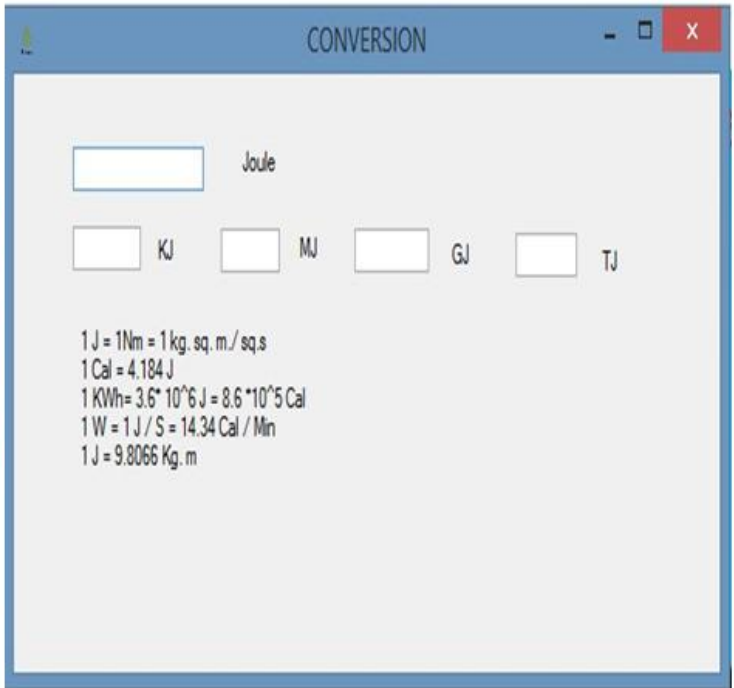

Figure 14 'Project' Window

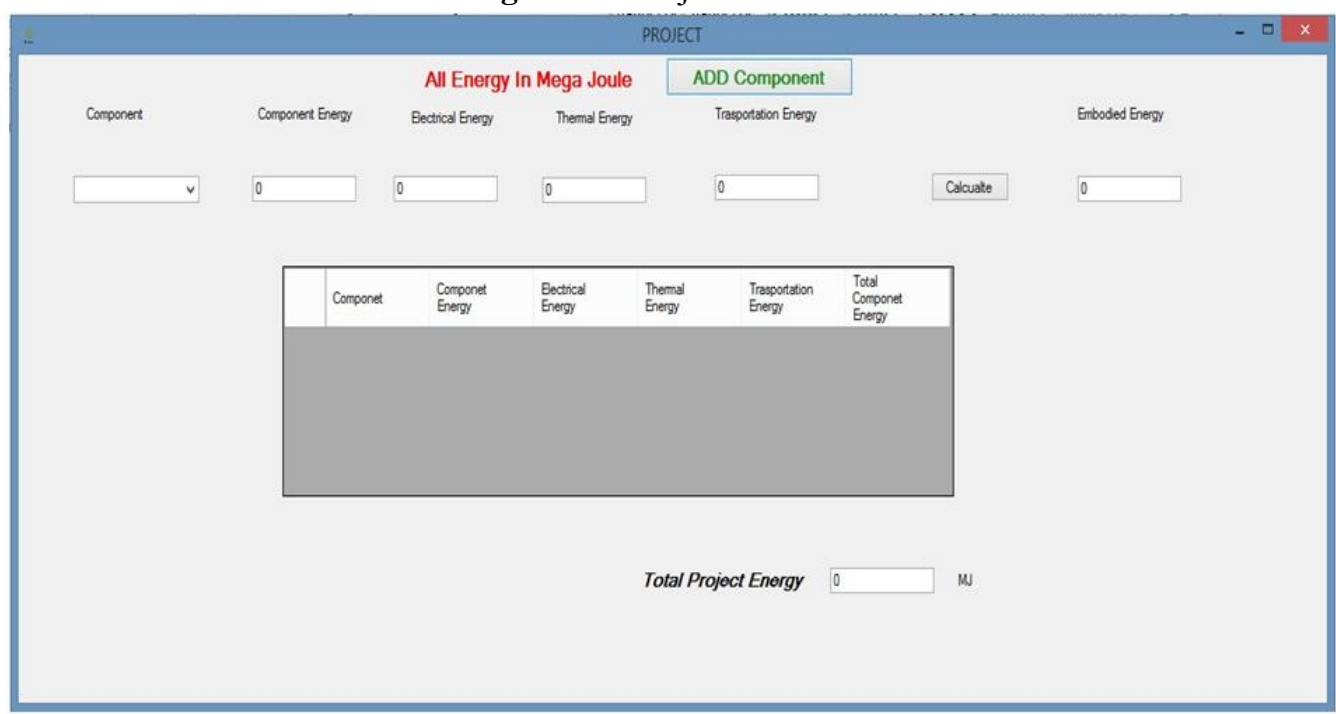


Figure 15 ‘Conversion’ Window

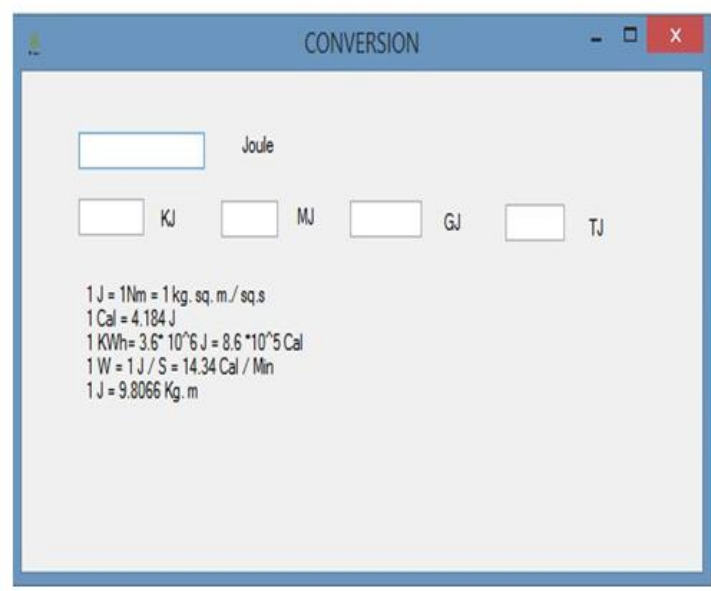

Figure 17 'How to use' Window

Components
1. Enter the component quantity.
2. Enter the proportion of material or efficiency.
3. Calculate embodied energy of component.
Types of Energy
1. Enter the quantity.
2. Enter Time, Efficiency or amount of material.
3. Calculate selected type of energy.
Project
1. Select component.
2. Follow the steps of calculation of component energy.
3. Copy the resulted component energy and paste it in component energy field which is in
project form.
4. Enter Electrical, Thermal and Transportation energy of selected component.
5. Click on calculate, to calculate embodied energy of selected component.
6. Click on total embodied energy to calculate energy of total project.

Figure 16 'Other Information’ Window

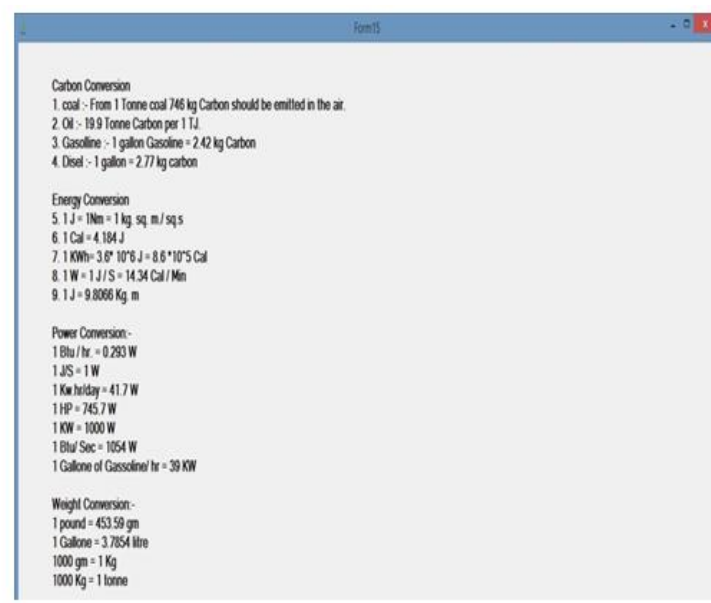

Figure 18 'Energy of Materials' Window

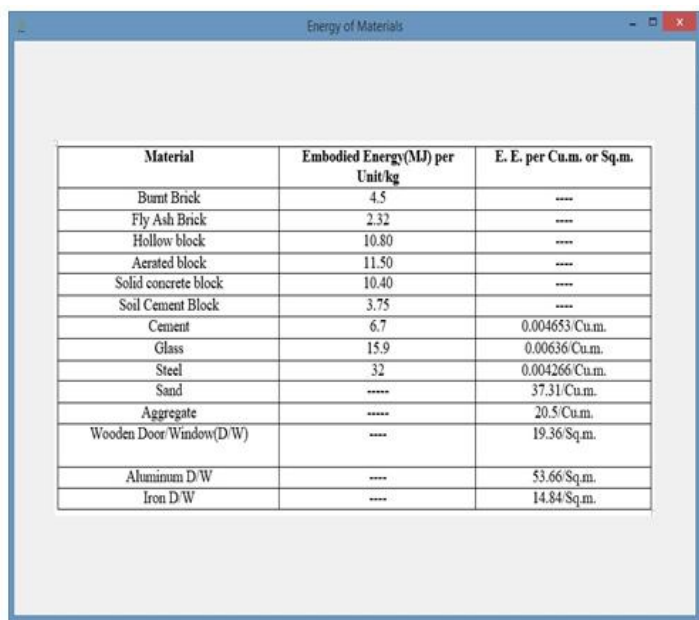

Figure 19 'Add Item’ Window

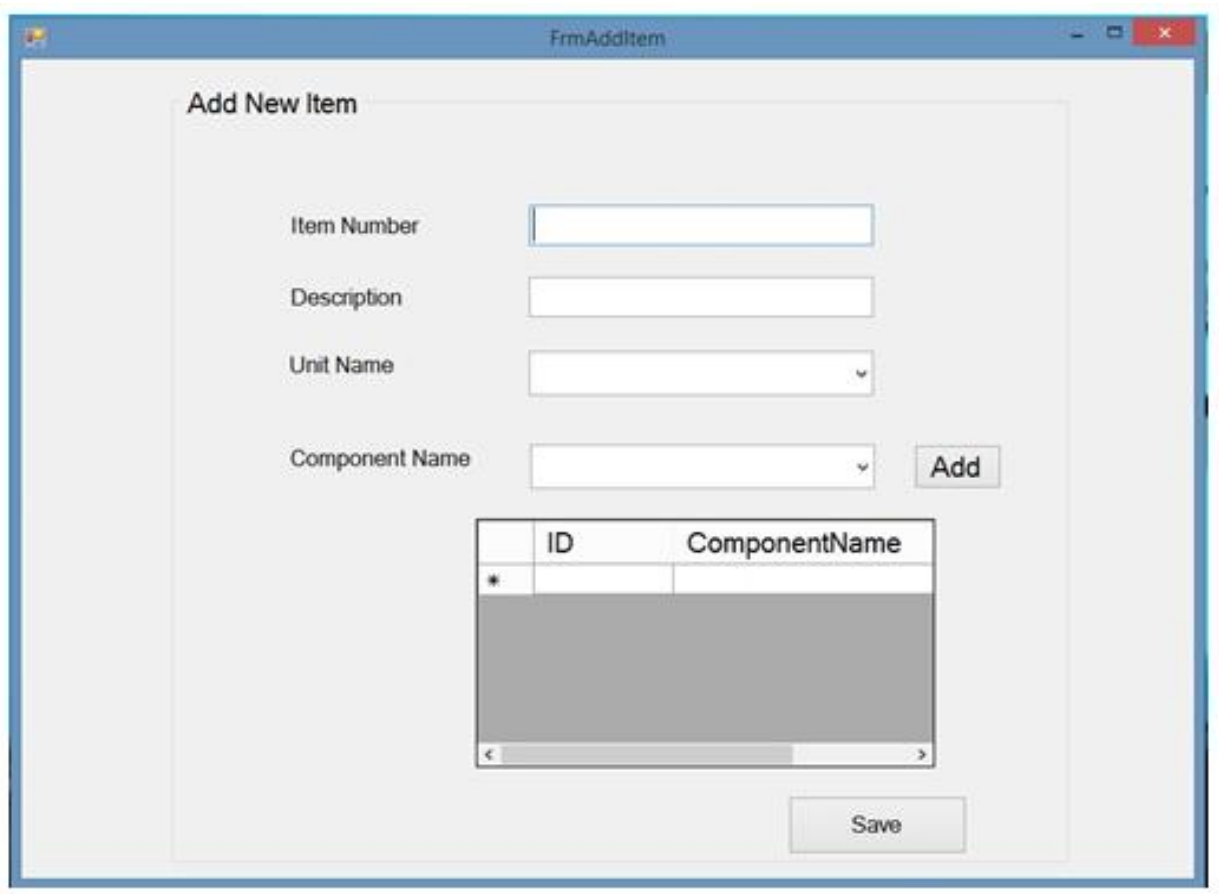


Figure 20 'Add Project' Window

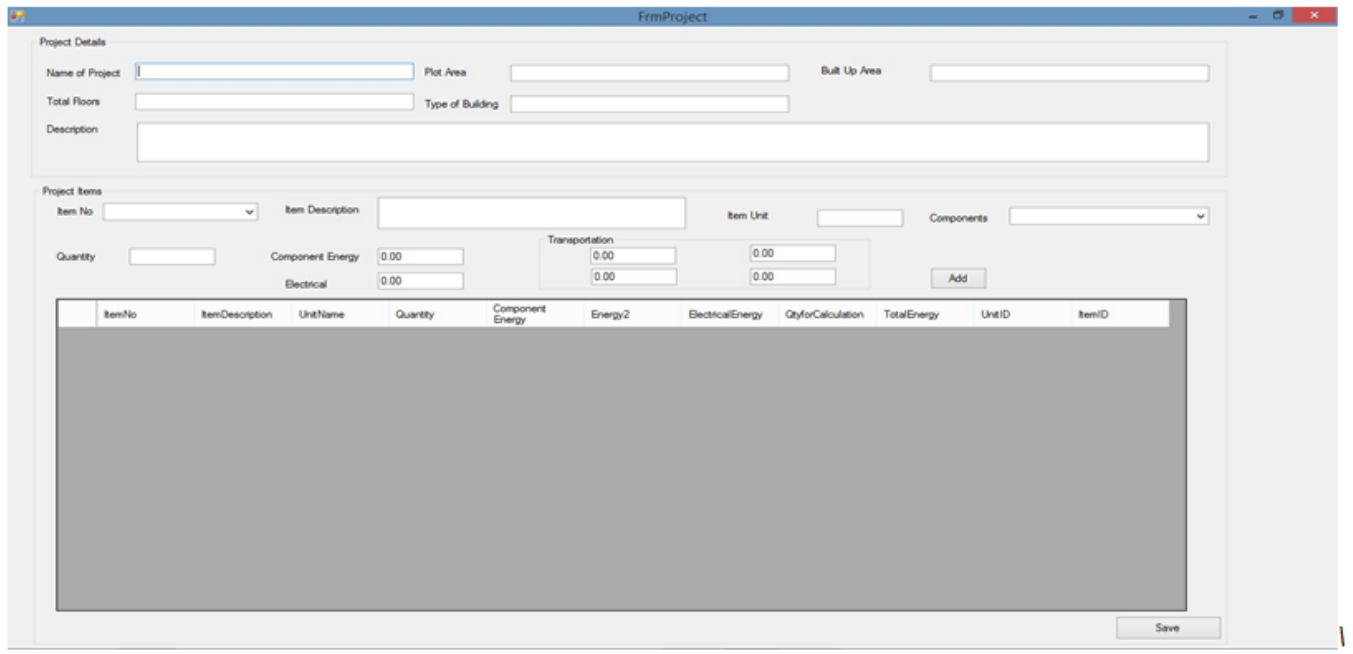

Figure 21 'Project List' Window

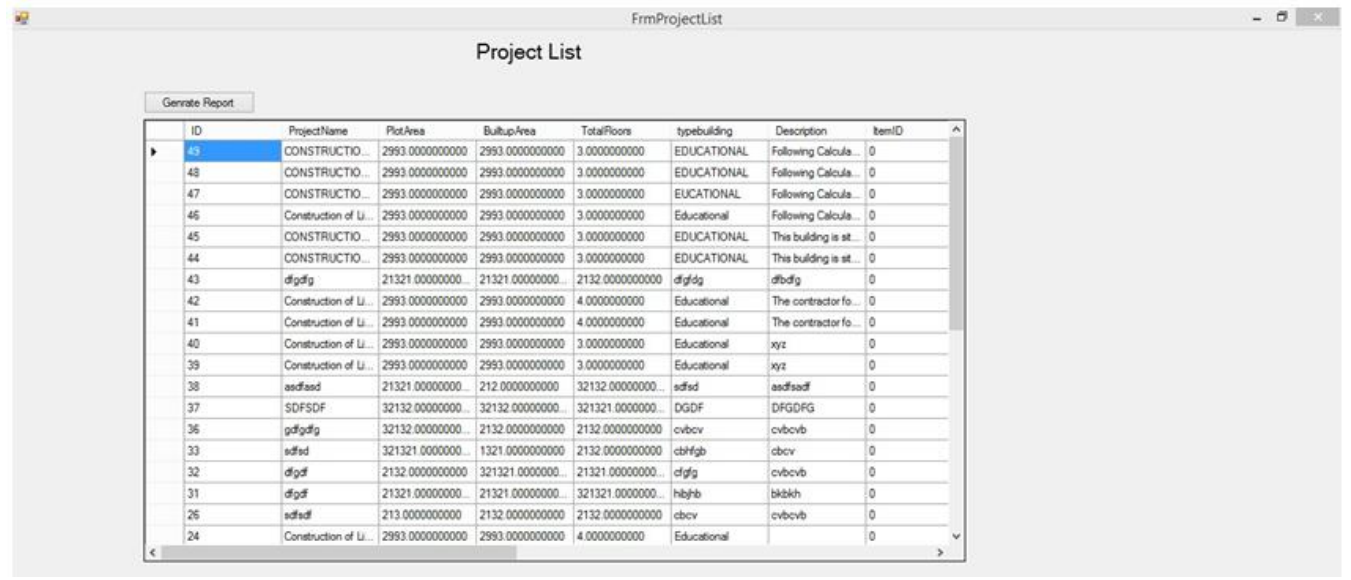

\section{Conclusion}

- More Accuracy can be achieved

- Less time is required

- Complex calculation can do easily

- More alternatives are available.

- The miscellaneous materials can be added.

- In 'project' window, infinite components can be added.

- New user can know about the conversion, E.E. of basic materials and general information about E.E.

\section{Reference}

[1]. Reddy B.V.V., (2004), "Sustainable Building Technologies", Current Science, vol 87, pp 899 - 907.

[2]. Reddy B.V.V. and Jagadish K.S., (2003), "Embodied energy of common and alternative building materials and technologies", Elsevier journal of Building and Environment, vol. 35, pp. 129-135.

[3]. Dixit Manish K., Fernández Jose L., LavySarel, Culp Charles H., (2012), "Need for an embodied energy measurement protocol for buildings: A review paper", Elsevier journal of Renewable and Sustainable Energy Reviews, vol. 16, pp. 3731-3735.

[4]. M. Kainuma, Y. Matsuoka, T. Morita, "Estimation of embodied CO2 emissions by general equilibrium model", European Journal of Operational Research (A part of Elsevier Team) 122 (2000) 392-404.

[5]. B.V. Venkatarama Reddy, P. Prasanna Kumar, "Embodied energy in cement stabilised rammed earth walls", Elsevier Journal of Energy and Buildings 42 (2010) 380-385. 